Article

\title{
Broadband Near-Infrared Absorber Based on All Metallic Metasurface
}

\author{
Ke Zhang ${ }^{1,2} \oplus$, Ruixiang Deng ${ }^{1,2}$, Lixin Song ${ }^{1,2, *}$ and Tao Zhang ${ }^{1,2, *}$ \\ 1 Key Laboratory of Inorganic Coating Materials CAS, Shanghai Institute of Ceramics, Chinese Academy of \\ Sciences, Shanghai 200050, China; zhangke@student.sic.ac.cn (K.Z.); dengrx@mail.sic.ac.cn (R.D.) \\ 2 Center of Materials Science and Optoelectronics Engineering, University of Chinese Academy of Sciences, \\ Beijing 100049, China \\ * Correspondence: 1xsong@mail.sic.ac.cn (L.S.); tzhang@mail.sic.ac.cn (T.Z.)
}

Received: 12 October 2019; Accepted: 29 October 2019; Published: 30 October 2019

check for updates

\begin{abstract}
Perfect broadband absorbers have increasingly been considered as important components for controllable thermal emission, energy harvesting, modulators, etc. However, perfect absorbers which can operate over a wide optical regime is still a big challenge to achieve. Here, we propose and numerically investigate a perfect broadband near-infrared absorber based on periodic array of four isosceles trapezoid prism (FITP) unit cell made of titanium (Ti) over a continuous silver film. The structure operates with low quality $(\mathrm{Q})$ factor of the localized surface plasmon resonance (LSPR) because of the intrinsic high loss, which is the foundation of the broadband absorption. The high absorption of metal nanostructures mainly comes from the power loss caused by the continuous electron transition excited by the incident light inside the metal, and the resistance loss depends on the enhanced localized electric field caused by the FITP structure. Under normal incidence, the simulated absorption is over $90 \%$ in the spectrum ranging from $895 \mathrm{~nm}$ to $2269 \mathrm{~nm}$. The absorber is polarization-independent at normal incidence, and has more than $80 \%$ high absorption persisting up to the incident angle of $\sim 45^{\circ}$ at $\mathrm{TM}$ polarization.
\end{abstract}

Keywords: metamaterial; broadband absorber; near-infrared; localized surface plasmon resonance

\section{Introduction}

In 2008, Landy et al. [1] first experimentally demonstrated the perfect metamaterial absorber, which works in microwave frequency band. Since then, many works concentrate on metamaterial absorber operating at frequency from microwave range [2,3] through terahertz [4,5], infrared [6,7], and into the visible region [8-10]. In the optical region, perfect metamaterial absorber has been an important branch of optical devices for its unique performance. It has undergone a rapid and flourishing development, finding a wide variety of potential application including thermal emitters [6,11], bolometers [12], solar energy harvesting [8,13], photodetections [7], and sensing [9,14,15]. The typical structure of metamaterial absorber is metal-dielectric-metal (MDM) structure $[6,7,16]$, consisting of patterned subwavelength periodic metal arrays on top of the central dielectric layer, backed by a metallic ground plane. Beyond that, some absorbers composed of plasmonic nanostructure/nanoparticles $[14,17,18]$ and metasurfaces [19-26] structure have been proposed and studied extensively. The absorption enhancement of incident light is derived from various types of electromagnetic (EM) resonances, such as propagating surface plasmon resonance (PSPR) [6], localized surface plasmon resonance (LSPR) [23], surface lattice resonance (SLR) [19,26], and so on. The perfect absorption is usually a single or multiple narrow band because of their nature of the resonances. However, the perfect broadband absorption is highly required for applications in the fields of energy harvesting and thermal emission. 
To achieve a large absorption bandwidth there are two common and direct strategies [27]. One method is planar arrangement [28,29], which requires to create multiple resonances in each unit cell via tailoring the size of the patterns on the top layer. In this case, multiple resonances $[8,30]$ will appear and perfect absorption can be achieved by incorporating resonators into a unit cell. Specifically, if these resonance absorption peaks are close enough, they will overlap with each other to form broadband absorption. The second method is vertical arrangement [31-33], which can lead to broadband absorption by stacking multiple top layers in the vertical direction. One can use different dielectrics and metals as an individual bilayer and stack these bilayers to form a consecutive broadband absorption spectrum by tuning the geometric parameters simultaneously. However, for the method one, the number of the resonators is restricted because of the competition of neighboring resonances, and the absorption performance is sensitive to the structure size, thus its absorption intensity and bandwidth expansion are limited. For method two, the broadband absorption performance can be improved further by increasing the number of metal/dielectric layers. Nonetheless, it is rather difficult to realize experimentally since they require precise control in complex fabrication processes, which also limit their potential applications. Therefore, finding a simple and effective strategy to achieve perfect broadband absorber is a challenging and significant task.

Metasurfaces are two dimensional arrays formed by subwavelength periodic nanostructures of metal and dielectric. This structure can easily localize the incident light and enhance absorption because of its effect of localized surface plasmon resonance. However, the existing researches $[10,19,21,23,26]$ mainly use noble metal or dielectric materials as the metasurface to achieve high absorption, which is difficult to achieve broadband absorption. High loss materials can excite low-Q surface plasmon resonances (SPRs), which is conducive to broadband absorption [34,35]. Therefore, we consider using high loss materials to design appropriate metasurface structure, aiming to widen the absorption peaks and get the perfect broadband absorber.

In this article, we propose a broadband and polarization-independent absorber based on simple and all metallic metasurface in near-infrared region. The structure consists of metallic array made of four isosceles trapezoid prism (FITP) unit cell over a continuous metal film. The absorption mechanism is explored through the analysis of the electric field distributions and the power loss profiles. Moreover, the absorption performance is numerically researched when the materials of the ground plane and the nanostructures are replaced by the other metal materials, respectively. For absorption spectrum, the effect of the geometric parameters of the unit cell structure on the absorption bandwidth is studied. Additionally, numerical simulations show the properties of the polarization angle and incident angle.

\section{Structure Design and Simulations}

As shown in Figure 1, the proposed broadband absorber is composed of metallic array made of FITP unit cell backed with a continuous metallic film on a silicon substrate. The optimized geometric parameters of the broadband absorber are: $\Lambda=800 \mathrm{~nm}, L=700 \mathrm{~nm}, w=150 \mathrm{~nm}, h=280 \mathrm{~nm}, t=100 \mathrm{~nm}$. It should be noticed that the metallic FITP structure is deformed from a metallic square ring structure. It is expected that the FITP structure can give rise to stronger resonance mode so as to result in a continuous strong broadband absorption in near-infrared region [36]. 


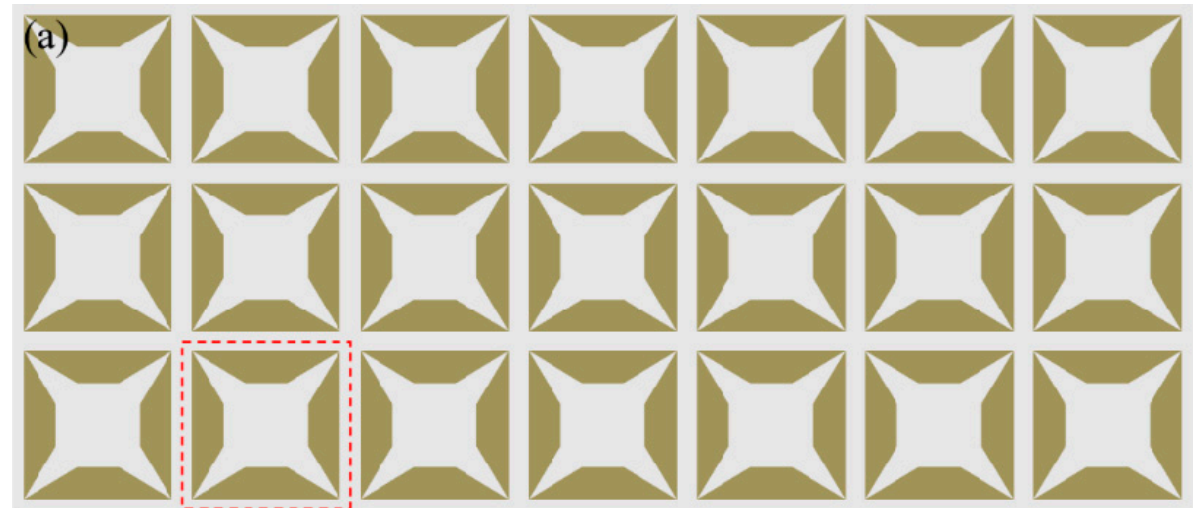

(b)

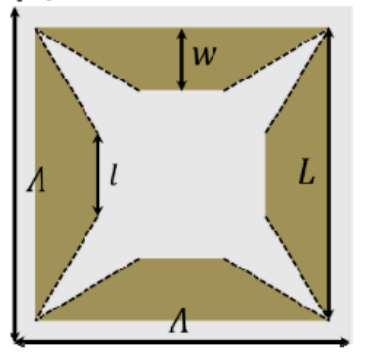

(c)

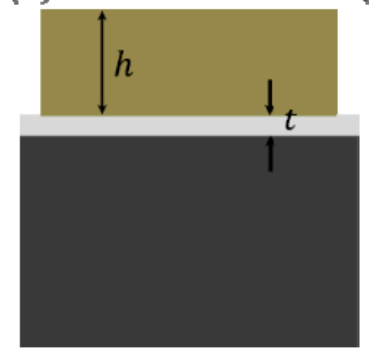

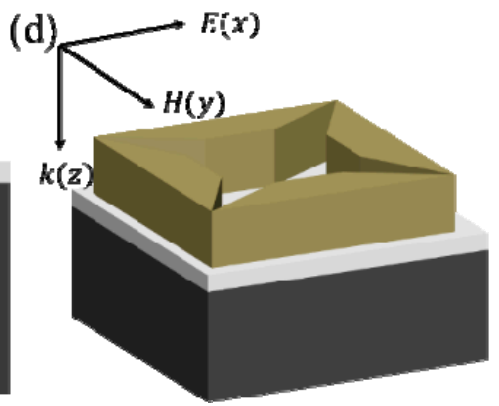

Figure 1. Schematic illustration of the proposed broadband absorber: (a) two-dimensional (2D) nanostructure array; (b-d) front, lattice, and perspective views of the unit cell nanostructure.

To investigate its absorption properties and mechanisms of the proposed absorber, full wave simulation was performed by a finite-different time-domain (FDTD) method based on commercial software (FDTD Solutions, Lumerical, Canada). In simulation, the bottom metallic layer is selected as silver (Ag) [37] film, the thickness of which is $100 \mathrm{~nm}$, much larger than the typical skin depth in near-infrared regime. Since the incident light cannot go through Ag film, the absorption can be calculated by $A(\lambda)=1-R(\lambda)$, where $R(\lambda)$ is the reflection as functions of wavelength $\lambda$. Titanium (Ti) [37] is used to composed the metasurface because of its priority in features of high loss and stability in optical region. In addition, we will explore the effect of using other different metal materials (such as gold $(\mathrm{Au})$, aluminum $(\mathrm{Al})$, chromium $(\mathrm{Cr})$, nickel $(\mathrm{Ni})$, tungsten $(\mathrm{W}))$ [37] as metallic ground plane and nanostructures on the absorption performance of the absorber in Section 3. The complex dielectric constant of all metals is fitted by a Drude-Lorentz model (with 6 coefficients) in the FDTD solutions. In simulation process, periodic boundary conditions were performed on unit cell in the $\mathrm{x}$ and $\mathrm{y}$ plane to represent the behavior of an infinite array at normal incidence. The structure is illuminated by an incident light with electric field parallel to the x-axis. For oblique angles, Bloch boundary conditions were performed on unit cell in the $\mathrm{x}$ and $\mathrm{y}$ plane. In both cases, a perfect matching layer was used in the $\mathrm{z}$ direction.

\section{Results and Discussions}

As mentioned above, the metallic FITP structure is deformed from a metallic square ring structure. To be specific, we can split the square ring into four isosceles trapezoids and get the proposed structure by decreasing the length of the short-side $(l)$. Figure 2 shows the simulated 2D absorption color map with the short-side decreasing from $400 \mathrm{~nm}$ to $0 \mathrm{~nm}$. We can see that these structures have similar absorbing behavior with two peaks and one dip (the white dotted line in Figure 2). The absorption performance of the square ring structure is poor because its absorption spectrum has a deeper dip. However, when just decreasing the length of the short-side, the absorption performance of the structure becomes better. For quantitative comparison of absorption performance, the relative bandwidth of the absorber [6], defined as $R B W(\geq 90 \%)=2 \times\left(\lambda_{l}-\lambda_{s}\right) /\left(\lambda_{l}+\lambda_{s}\right)$, where $\lambda_{l}$ and $\lambda_{s}$ are the long and short 
limits of a wavelength range with absorption above $90 \%$, respectively. Therefore, we can find that the $R B W(\geq 90 \%)$ first increases and then decreases as the short-side length decreases. When the short-side length is smaller than $275 \mathrm{~nm}$, the $R B W(\geq 90 \%)$ of absorbers can reach above $80 \%$. Furthermore, the designed absorber has best absorption performance which is greater than $90 \%$ from $895 \mathrm{~nm}$ to $2269 \mathrm{~nm}$ and the $R B W(\geq 90 \%)$ reaches as high as $86.9 \%$, when the short-side length is $275 \mathrm{~nm}$.

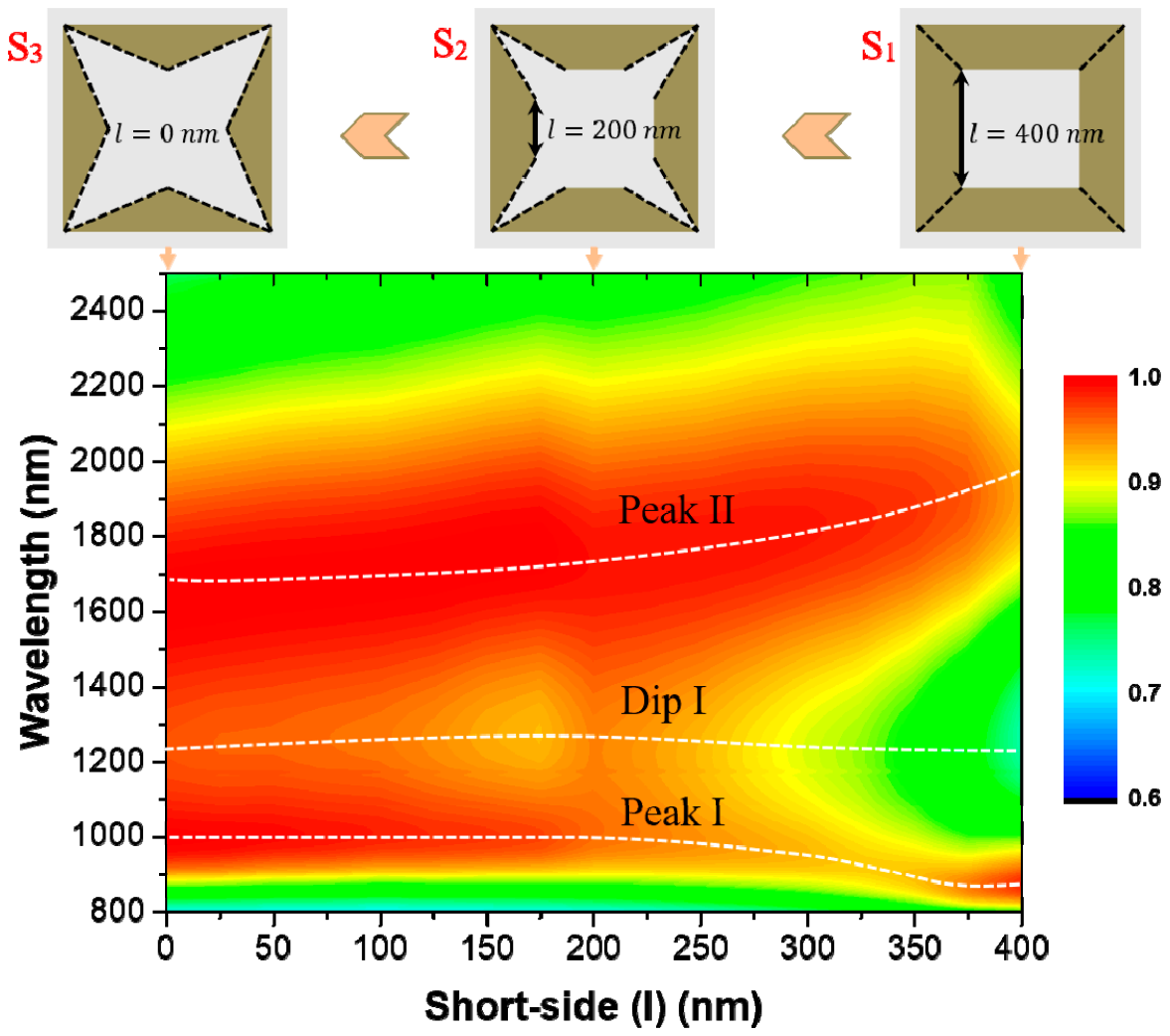

Figure 2. The simulated 2D absorption $(A(\lambda))$ color map as functions of the short-side length $(l)$ and the wavelength. Parameters: $\Lambda=800 \mathrm{~nm}, L=700 \mathrm{~nm}, w=150 \mathrm{~nm}, h=280 \mathrm{~nm}, t=100 \mathrm{~nm}$.

To reveal the working mechanism behind the stronger broadband absorption, we comparatively studied the electric field distributions of the three structures $S_{1}, S_{2}$, and $S_{3}$ (the plane structure is illustrated in Figure 2, corresponding length of short-side are $400 \mathrm{~nm}, 200 \mathrm{~nm}$ and $0 \mathrm{~nm}$, respectively) at different absorption peaks and dips. Figure $3 \mathrm{~b}-\mathrm{d}$ shows the electric field distributions of the structure $\mathrm{S}_{1}$. For peak $P_{1}$ the localized electric fields are concentrated at the inner edge while it locates at the outer edge for peak $P_{2}$. Obviously, the two absorption peaks originate from the excitation of the LSPR $[6,23,26]$. Since $\mathrm{Ti}$ is dispersive and has a relatively large imaginary part, the intrinsic absorption coefficient of Ti is very big, resulting in a rather low Q-factor for broadened absorption bandwidth [6,38]. However, at the $\operatorname{dip} D$, the electric field is distributed at both sides of the square ring, and the electric field intensity is lower than that of the peaks, which leads to a poor absorption.

When the length of the short-side is decreased to $200 \mathrm{~nm}$, the square ring structure becomes the four isosceles trapezoid structure $\left(S_{2}\right)$. As shown in Figure 3e, the intensity of the dip and the two peaks all exceeds $90 \%$, indicating better absorption property of the structure $S_{2}$. According to Figure $3 \mathrm{f}-\mathrm{h}$, the localized electric field mainly concentrates on the short-side at the peak $P_{1}{ }^{\prime}$. It suggests that the absorption intensity at $P_{1}{ }^{\prime}$ will be lower than that at $P_{1}$, agreeing with the absorption spectrum. Different from the structure $S_{1}$, E-field of structure $S_{2}$ is distributed at the corner and the inner side at the dip $D^{\prime}$, while the E-field is mainly localized in the corner at the peak $P_{2}{ }^{\prime}$. The absorption intensity at dip $D^{\prime}$ and peak $P_{2}{ }^{\prime}$ both increase because of a stronger localized electric resonance result from smaller length of the short-side. 
Finally, when the length of short-side is decreased to zero, we can get the structure $S_{3}$. As shown in Figure 3i, broadband and high absorption is achieved, but the bandwidth is slightly reduced relative to the structure $S_{2}$. We can notice that the absorption intensity exceeds $99 \%$ at peaks $P_{1}{ }^{\prime \prime}$ and $P_{2}$ ", and exceeds 95\% at dip $D^{\prime \prime}$. As shown in Figure 3j-1, the electric fields can be better concentrated at the corner in $\mathrm{S}_{3}$, which demonstrates the collective oscillations of conductive electrons, evidently suggesting the existence of the LSPR mode [16]. In addition, the electric fields of $S_{3}$ are much stronger than that of $S_{1}$ and $S_{2}$, proving that structure $S_{3}$ can enhance the electric fields [36]. In this manner, structure $S_{3}$ can also achieve broadband absorption based on the stronger localized electric field and low-Q LSPR.

(a)

(e)

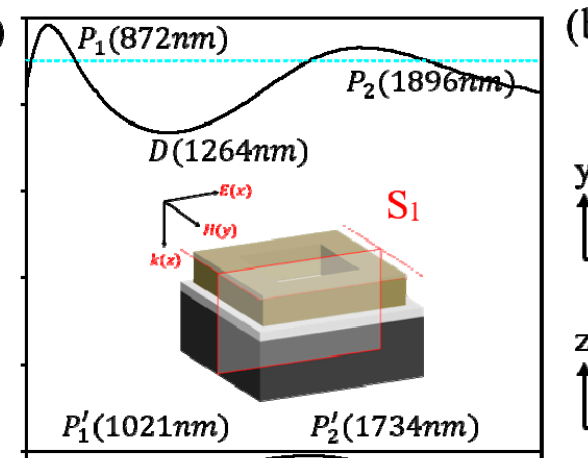

(b)

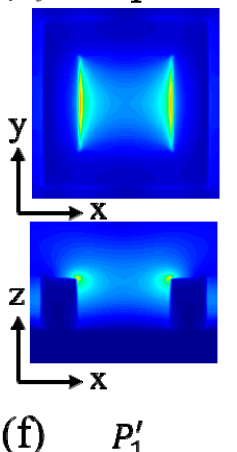

(f)

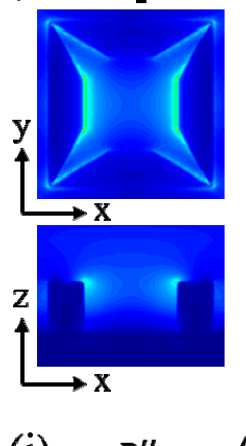

(j)

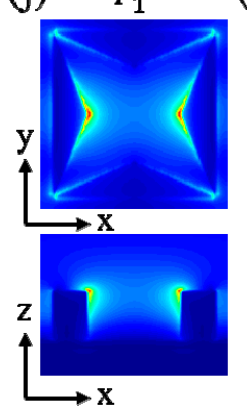

(c)
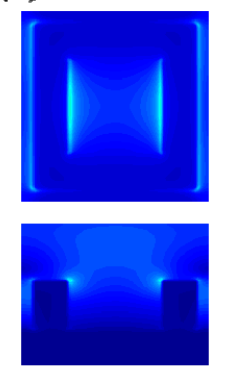

(g) $\quad D^{\prime}$
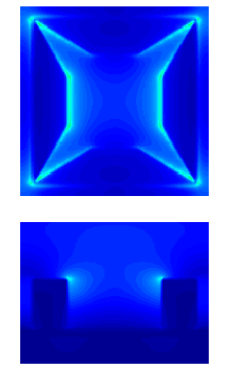

(k)

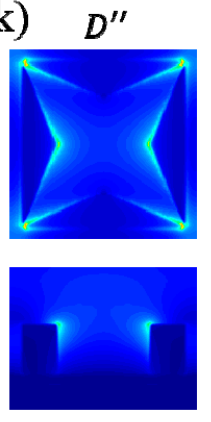

(d) $\quad P_{2}$

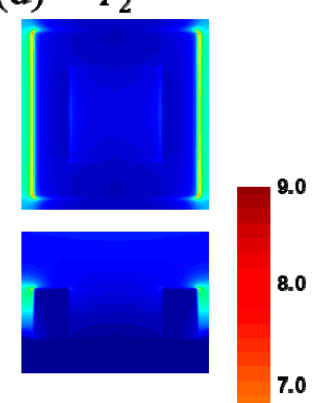

(h) $P_{2}^{\prime}$
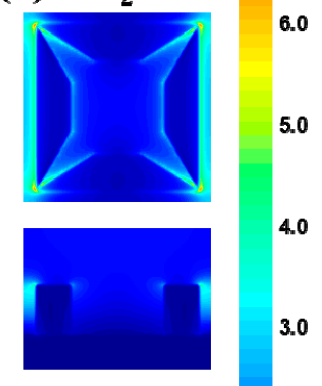

(1)

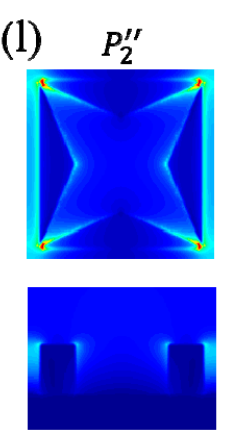

Figure 3. Absorption spectrum and electric field intensity distributions of the three structures at normal incidence. (a,e,i) Absorption spectrum of the structure $S_{1}, S_{2}$, and $S_{3}$. (b-d) The electric field distributions of $S_{1}$ at $P_{1}, D$, and $P_{2}$. (f-h) The electric field distributions of $S_{2}$ at $P_{1}{ }^{\prime}, D^{\prime}$, and $P_{2}{ }^{\prime}$. (j-l) The electric field distributions of $S_{3}$ at $P_{1}{ }^{\prime \prime}, D^{\prime \prime}$, and $P_{2}{ }^{\prime \prime}$.

To further and directly study the physical mechanism, we also studied the power loss distributions $[19,23,39]$ of the three structures at the dip and two peaks. In this structure, the power loss comes from the resistance loss $(q)$, which can be expressed by [40],

$$
q=\frac{1}{2} \varepsilon_{0} \omega \varepsilon_{m}^{\prime \prime}|E|^{2}
$$


where $\varepsilon_{0}$ is the vacuum dielectric constant, $\omega$ is the angular frequency of light, $\varepsilon_{m}$ " is the imaginary part of the relative permittivity of a metal $\left(\varepsilon_{m}=\varepsilon_{m}{ }^{\prime}+\varepsilon_{m}{ }^{\prime \prime}\right)$, and $E$ is the intensity of localized electric field. According to the Equation (1), the cross-section colormaps of the power loss are calculated and shown in Figure 4.

From profiles of the power loss in Figure 4, we can see that the power loss is mainly concentrated in Ti nanostructures. Power is absorbed at the corner and throughout inner edge region of the Ti nanostructures, which is consistent with the distributions of the electric field. By comparing Figures 3 and 4, we can find that the variation trends of power loss intensity are in agreement with that of the absorption spectra from $S_{1}$ to $S_{3}$. In other words, the absorption magnitude of incident light is significantly enhanced when the structures change from $S_{1}$ to $S_{3}$. It supports the discussion that decreasing the short-side of the structure can enhance the localized electric field and result in the high absorption performance of Ti nanostructures.

(a)

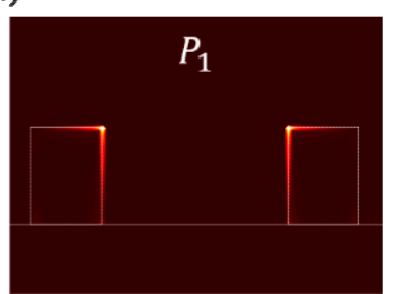

(d)

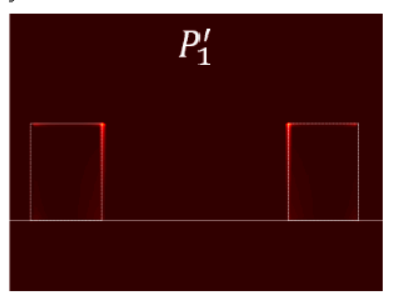

(g)

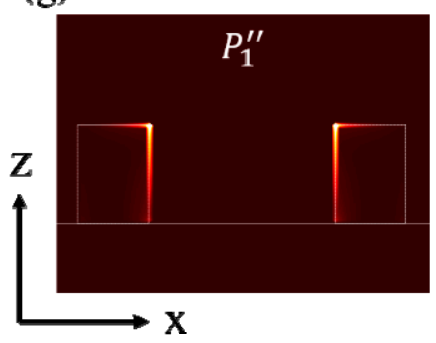

(b)

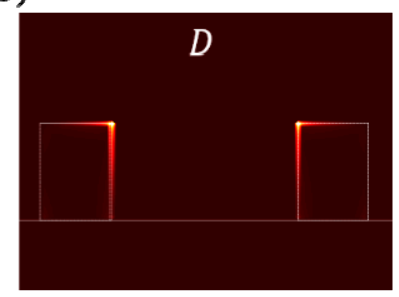

(e)

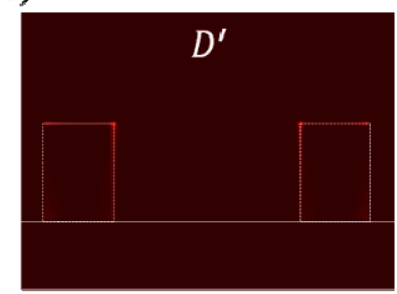

(h)

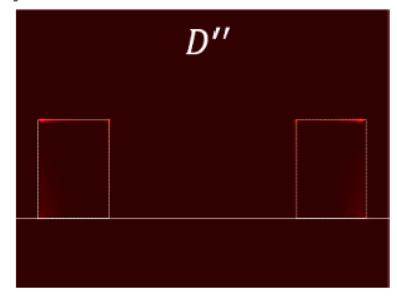

(c)

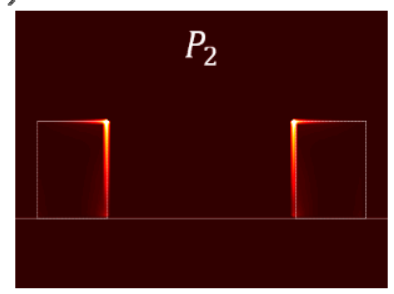

(f)

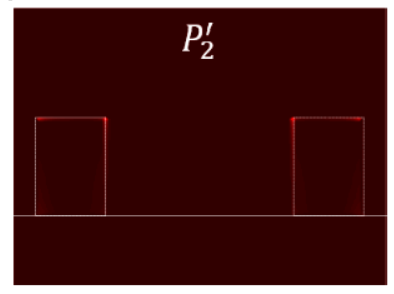

(i)

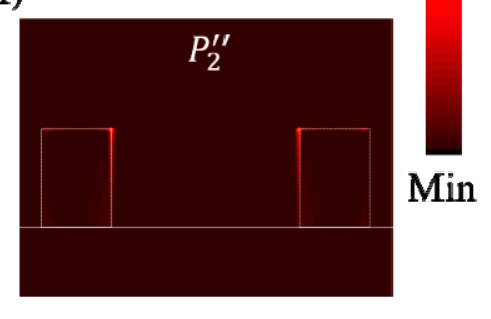

Figure 4. The cross-section colormaps describe the normalized power loss in the three structures. Structure $S_{1}$ at (a) $P_{1}$, (b) $D$, and (c) $P_{2}$. Structure $S_{2}$ at (d) $P_{1}{ }^{\prime},(\mathbf{e}) D^{\prime}$, and (f) $P_{2}{ }^{\prime}$. Structure $S_{3}$ at $(\mathbf{g}) P_{1}{ }^{\prime \prime}$, (h) $D^{\prime \prime}$, and (i) $P_{2}{ }^{\prime \prime}$. Brighter areas correspond to higher power loss. The position of the cross-section is consistent with Figure 3.

We analyzed the effects of different types of composed metal material on absorption performance. First, $\mathrm{Ti}$ is used as the nanostructures, and $\mathrm{Ag}$ ground plane is replaced by metal materials ( $\mathrm{Au}, \mathrm{Al}, \mathrm{Cr}$, $\mathrm{Ni}, \mathrm{W}$, and $\mathrm{Ti}$ ), the contrastive absorption spectra are shown in Figure 5a. We can see that only the absorption bandwidth changes slightly in this case. Next, using Ag as the ground plane and choosing metal materials ( $\mathrm{Ag}, \mathrm{Au}, \mathrm{Al}, \mathrm{Cr}, \mathrm{Ni}$, and $\mathrm{W}$ ) to replace the Ti nanostructures, the contrastive absorption spectra are shown in Figure 5b. We can observe that using $\mathrm{Cr}, \mathrm{Ni}, \mathrm{W}$, and $\mathrm{Ti}$ as the nanostructures, broadband absorption can be achieved and only the absorption bandwidth changes a little. Differently, using $\mathrm{Ag}, \mathrm{Au}$, and $\mathrm{Al}$ as the nanostructures cannot achieve broadband absorption, and there are only a few weak absorption peaks at the resonant frequency. In addition, using $\mathrm{Ti}$ and Ag as the nanostructures, we calculate the proportion of light absorbed by the surface metal pattern and the 
bottom metal film, as shown in Figure $5 c$,d. It indicates that the light is absorbed mainly by the surface metal pattern. Which is also consistent with the result of the power loss profiles shown in Figure 4.
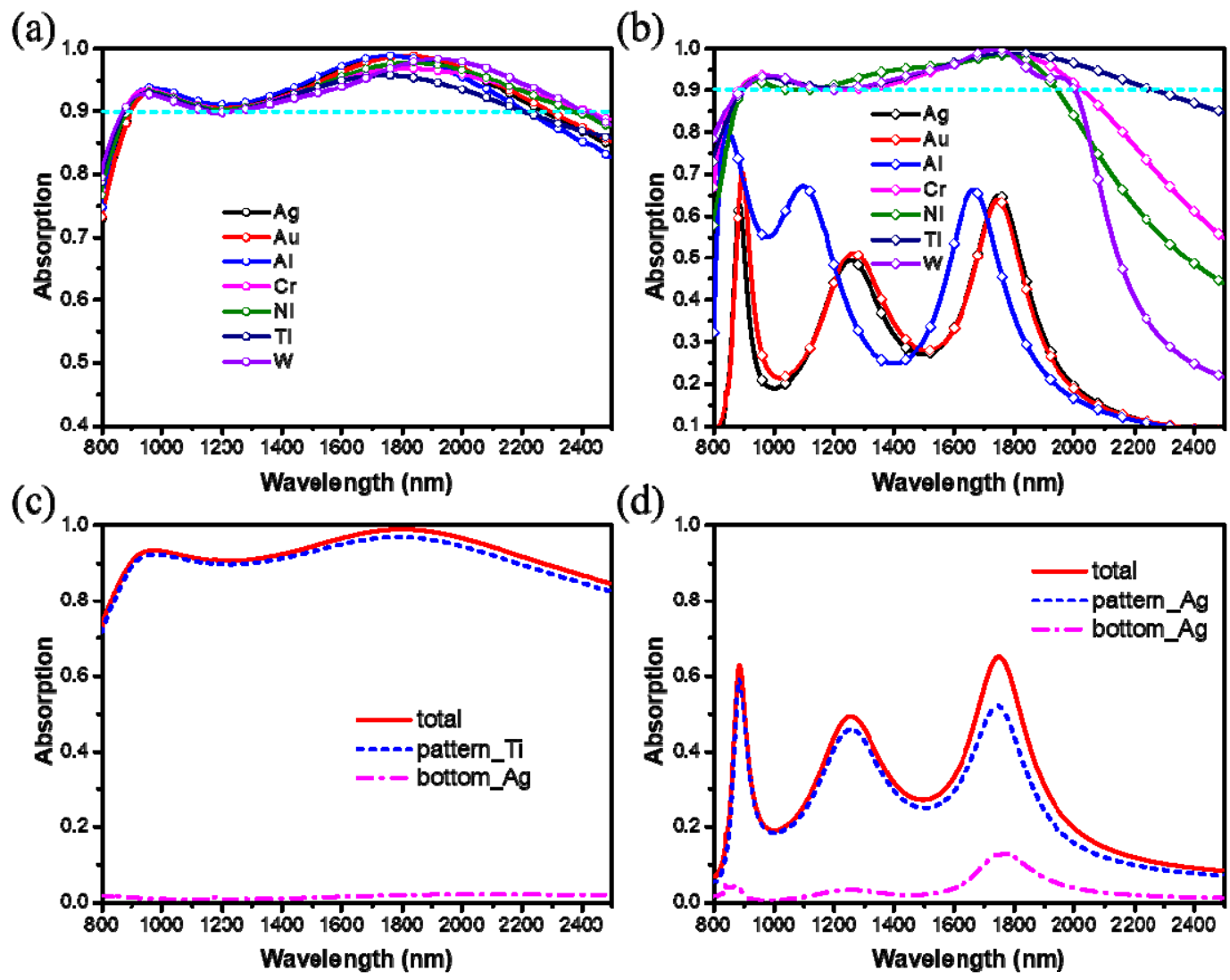

Figure 5. The contrastive absorption spectra of the broadband absorber when (a) metallic ground plane and (b) metallic nanostructures are replaced by the other metal materials. The contrastive absorption spectra of the total, pattern and bottom of (c) Ti-Ag structure and (d) Ag-Ag structure. Parameters: $\Lambda=800 \mathrm{~nm}, L=700 \mathrm{~nm}, w=150 \mathrm{~nm}, h=280 \mathrm{~nm}, t=100 \mathrm{~nm}, l=275 \mathrm{~nm}$.

In order to clearly understand the primary cause why different types of metal materials lead to different absorption characteristics in the designed absorber, we use the Hagen-Rubens (H-R) relation [41] to describe the reflection and absorption performance of the metal film. The reflection $(R)$ of metals at normal incidence can be derived by extended H-R relation [42,43],

$$
R=1-\frac{4 n}{(n+1)^{2}+k^{2}}
$$

where $n$ and $k$ are the real and imaginary parts of the refractive index of the metal. Figure 6a shows the calculated reflection spectra of the metal materials by the Equation (2). We can observe that most of the incident light is reflected ( $k$ is much more than $n$ ), and the rest is absorbed for the metals of $\mathrm{Ag}$, $\mathrm{Au}$, and $\mathrm{Al}$. However, the reflection of the incident light reduces ( $n$ is close to $k$ ) for the metals of $\mathrm{Cr}$, $\mathrm{Ni}, \mathrm{W}$, and $\mathrm{Ti}$, which is to say they have intrinsic high absorption, especially in the short wavelength. In addition, the mechanism which the incident light is absorbed by the metal depends on that the Fermi level in metals locates inside a continuous energy band. Consequently, light that enters a metal can be absorbed with a tiny propagating length (skin depth), usually less than $100 \mathrm{~nm}$ [44]. Therefore, these metal materials used as the ground plane are opaque and have little effect on the absorption performance of the designed absorber when they are thicker than skin depth, which is consistent with the result shown in Figure 5a. 
(a)

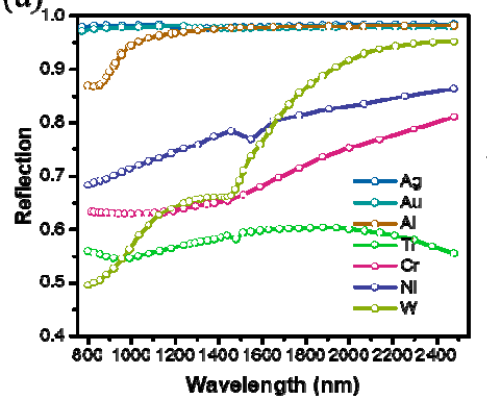

(b)

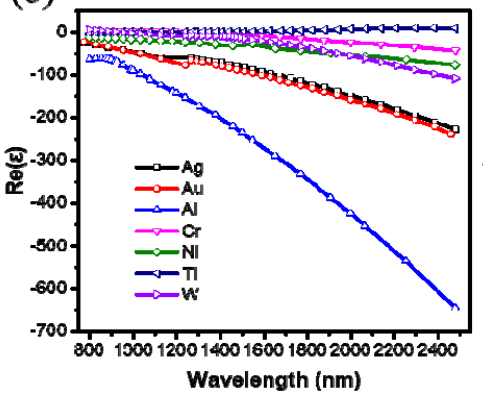

(c)

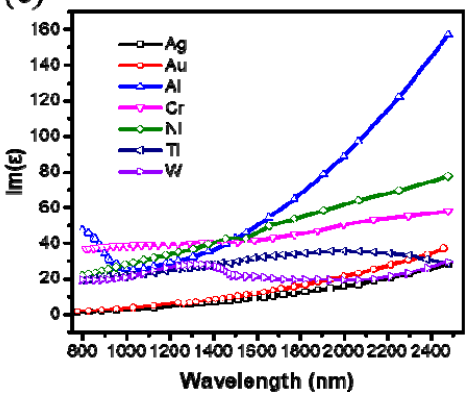

Figure 6. (a) The calculated reflection spectra of metal materials by the Equation (2). The real part (b) and imaginary part (c) of the permittivity of metal materials.

Accordingly, we can classify these metals into two types by their permittivity $\left(\operatorname{Re}(\varepsilon)=n^{2}-k^{2}\right.$ and $\operatorname{Im}(\varepsilon)=2 n k)$, as shown in Figure $6 \mathrm{~b}$,c. First, $\mathrm{Ag}, \mathrm{Au}$, and $\mathrm{Al}$ are the first type of metals, which have high reflection property because of having large real part of the permittivity. This characteristic can lead to weak absorption because of impedance mismatch even if it has a large imaginary part of the permittivity, as in the case of Al. This also explains why broadband absorber cannot be achieved by using $\mathrm{Ag}, \mathrm{Au}$, and $\mathrm{Al}$ as the nanostructures, as shown in Figure $5 \mathrm{~b}$. Then, $\mathrm{Cr}, \mathrm{Ni}, \mathrm{W}$, and $\mathrm{Ti}$ are the second type of metals, which is high loss metal materials because of having small real part and large imaginary part of the permittivity. Therefore, when they are used as the pattern layer of the absorber, it is easy to achieve impedance matching to decrease the reflection of the incident light. And strong localized electric field excited by the incident light, combining with high loss (high- $\varepsilon_{m}{ }^{\prime \prime}$ ) property of this metals can result in high resistance loss according to the Equation (1). Therefore, we can conclude that the high absorption of the designed absorber mainly comes from two parts. The first part is the power loss caused by the continuous electron transition excited by the incident light inside the metal. And the second part is the resistance loss mainly depends on the enhanced localized electric field caused by the nanostructures.

In addition, we investigate numerically the absorption properties of the designed broadband absorber under different geometric parameters of the unit cell nanostructure. As shown in Figure 7, we present the absorption spectra with four different parameters $(\Lambda, L, h, w)$, when changing only one parameter at a time, while keeping the other parameters unchanged. Similarly, through calculating the $R B W(\geq 90 \%)$ of the curves, we find that the bandwidth of the absorber is first increased and then decreased with the increase of the geometric parameters. Differently, each parameter has a different effect on the change of the absorption curve. Figure 7a shows that the period $(\Lambda)$ of unit cell may determine the long wavelength limit of $R B W(\geq 90 \%)$. As $\Lambda$ increases from $770 \mathrm{~nm}$ to $830 \mathrm{~nm}$, the long wavelength limit is blue-shifted, the magnitude of the dip increase, resulting in $R B W(\geq 90 \%)$ to increase and then decrease. From Figure $7 \mathrm{~b}$, although with the increase of the side length $(L)$ the intensity of the short wavelength resonance peak is enhanced, but the magnitude of the dip will decrease to less than 0.9 when the $L$ is bigger than $700 \mathrm{~nm}$. Thus, the $R B W(\geq 90 \%)$ is first increased and then decreased. Figure $7 \mathrm{c}$ shows the absorption efficiency as a function of the height $(h)$. It indicates that as the $h$ increases, the short and long wavelength limit and the position of two resonance peaks are red-shifted, but the intensity of the dip is reduced, which lead to first increase and then decrease of the $R B W(\geq 90 \%)$. Finally, the wire width $(w)$ has a more obvious effect on the absorption intensity at short wavelength. As the $w$ increases, the long wavelength limit is red-shifted, but the absorption intensity at the short wavelength decreases rapidly, as shown in Figure $7 \mathrm{~d}$. The maximum $R B W(\geq 90 \%)$ is found when the $w$ is $150 \mathrm{~nm}$. Therefore, the results of the bandwidth dependence on these parameters indicate that the optimized parameters are reasonable. In addition, the results of parametric analysis also indicate that the absorption properties can be tuned by varying the geometric parameters of the $\mathrm{Ti}$ unit cell nanostructure. 
(a)

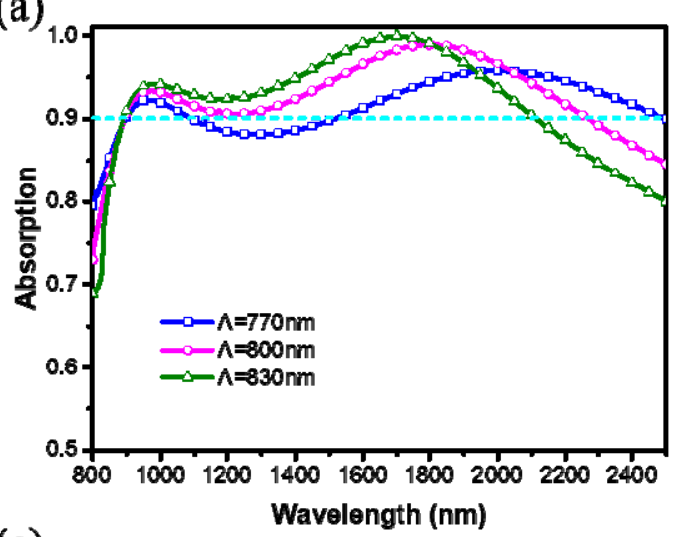

(c)

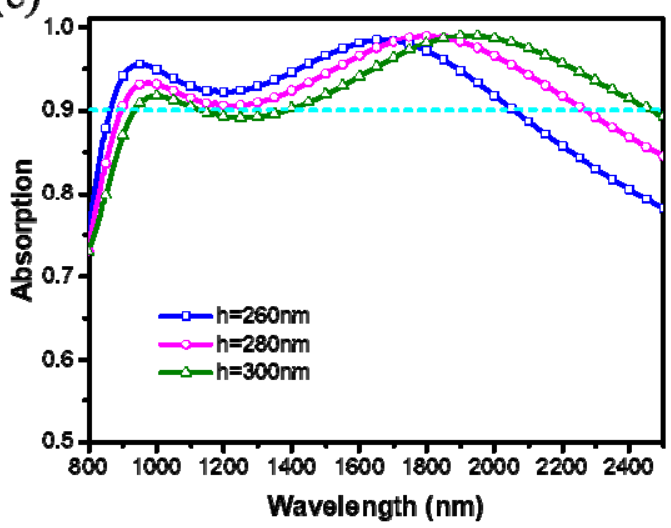

(b)

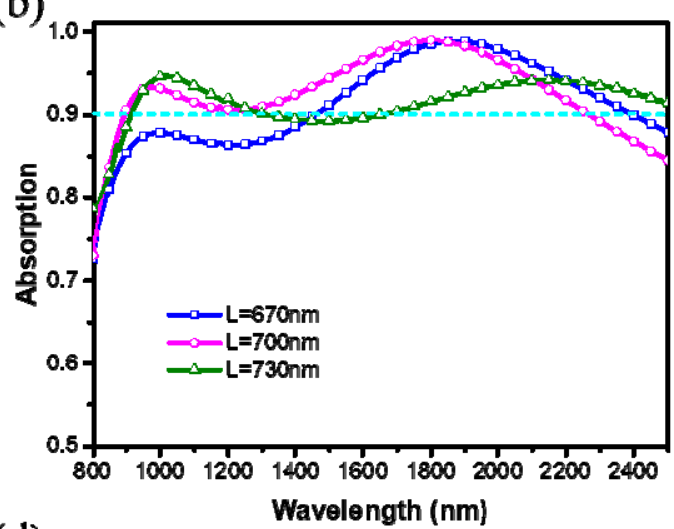

(d)

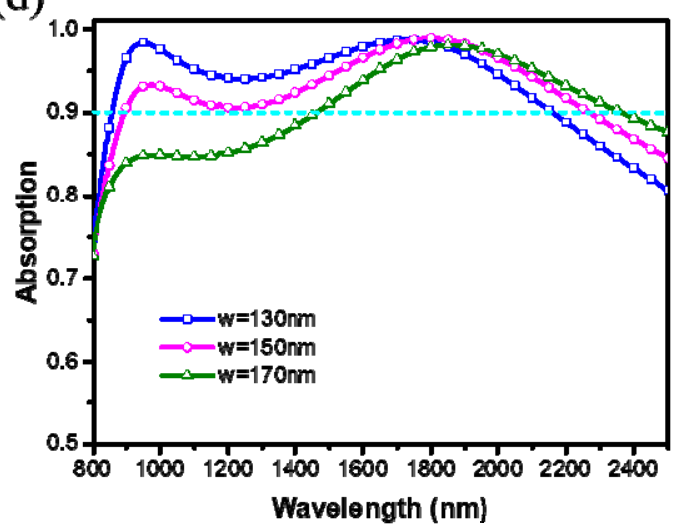

Figure 7. Simulated absorption spectra of the designed absorber under different geometric parameters: (a) period $(P),(\mathbf{b})$ side length $(L),(\mathbf{c})$ height $(h)$, and $(\mathbf{d})$ wire width $(w)$ of the unit cell nanostructure.

For practical application, the absorption properties should consider the effect of polarization angle and incident angles. The effect of polarization angle on the absorption spectra has been studied at the normal incidence, as shown in Figure 8a. The absorption behavior does not show any variation with different polarization angles, which change from $0^{\circ}$ to $90^{\circ}$. This is due to the high rotational symmetry of the unit cell nanostructure [38]. Furthermore, the absorption spectra of the absorber at oblique incidence have been calculated, the full-wave simulations are performed for both Transverse Electric (TE) polarization (the electric field of the incident light is parallel to $y$-axis) and Transverse Magnetic (TM) polarization (the magnetic field of the incident light is parallel to $y$-axis), as shown in Figure 8b,c. It is observed that obvious variation of the absorption spectrum happens at different oblique angles. In TE polarization, the absorption intensity drops obviously, and the $R B W(\geq 90 \%)$ decreases dramatically with the increase of the incident angle. It is because the electric resonance mode cannot be excited effectively when the incident angle gets larger. In other words, electrics fields at large incident angles are confined less efficiently in the pattern layer than at normal incidence, hence leading to a weaker absorption. In TM polarization, one can clearly see the absorption intensity drops at the short wavelength with the increase of incident angle from $0^{\circ}$ to $45^{\circ}$, which lead to a decrease of the $R B W(\geq 90 \%)$. However, the decrease of the absorption for TM mode is not obvious compared with the one for the TE mode, since the magnetic response plays a dominant role over the electric response for TM mode. Therefore, the absorption intensity at short wavelength decreases slightly, and the absorption magnitude maintains higher value than 0.8 in the whole wavelength range. Thus, the proposed broadband absorber is polarization-independent at normal incidence and incident-angle-sensitive at oblique incidence. But for TM polarization, the absorption intensity can still be greater than $80 \%$ at an incident angle of $45^{\circ}$. 
(a)

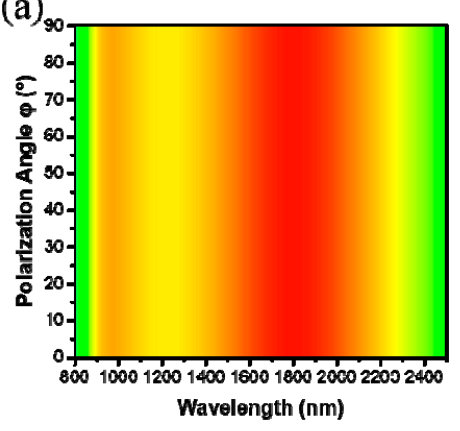

(b)

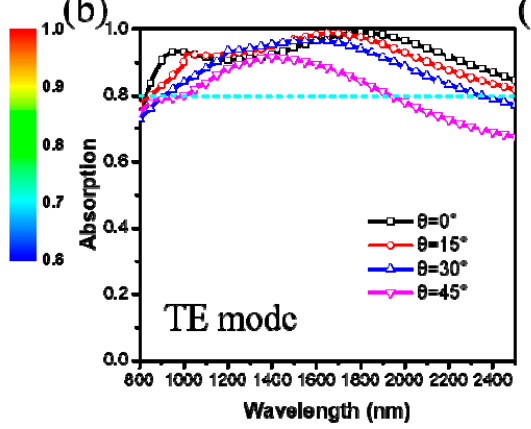

(c)

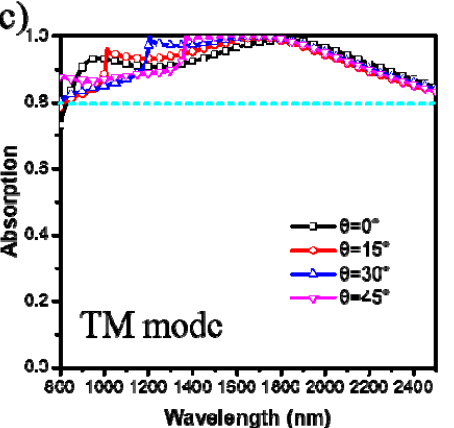

Figure 8. Simulated absorption as a function of (a) polarization angles $\varphi ;(\mathbf{b})$ and (c) incident angles $\theta$ with TE polarization mode and TM polarization mode. The parameters are the same as Figure 5.

\section{Conclusions}

In summary, we proposed a broadband and polarization-independent absorber based on simple and all metallic metasurface working in the near-infrared region. The structure consists of metallic FITP array over a continuous $\mathrm{Ag}$ film. Under normal incidence, the simulative absorption is over $90 \%$ in the spectrum ranging from $895 \mathrm{~nm}$ to $2269 \mathrm{~nm}$. Highly-efficient broadband absorption is ascribed to strong local effect of electric field and low-Q LSPR supported by Ti nanostructures. Specifically, through analyzing the electric field distributions, power loss profiles and reflection spectra of metal materials, we observe that the high absorption of the designed absorber mainly comes from the power loss caused by the continuous electron transition excited by the incident light inside the metal, and the resistance loss depends on the enhanced localized electric field caused by the FITP structure. For absorption spectrum, we can change the absorption bandwidth through tuning the geometric parameters of the nanostructures. Moreover, numerical simulations show that the broadband absorber is independent of the polarization angles at normal incidence, and has more than $80 \%$ high absorption persisting up to the incident angle of $\sim 45^{\circ}$ at TM polarization. Therefore, the broadband absorber may have potential applications in controllable thermal radiation and energy harvesting.

Author Contributions: Conceptualization, K.Z.; data curation, K.Z.; formal analysis, K.Z.; funding acquisition, T.Z. and L.S.; investigation, K.Z.; project administration, T.Z. and L.S.; resources, T.Z.; supervision, T.Z. and L.S.; validation, R.D.; visualization, K.Z.; writing—original draft, K.Z.; writing—review and editing, R.D., T.Z. and L.S.

Funding: This research received no external funding.

Conflicts of Interest: The authors declare no conflict of interest.

\section{References}

1. Landy, N.I.; Sajuyigbe, S.; Mock, J.J.; Smith, D.R.; Padilla, W.J. Perfect metamaterial absorber. Phys. Rev. Lett. 2008, 100, 207402. [CrossRef] [PubMed]

2. Shen, Y.; Zhang, J.; Pang, Y.; Zheng, L.; Wang, J.; Ma, H.; Qu, S. Three-Dimensional Resistive Metamaterial Absorber Loaded with Metallic Resonators for the Enhancement of Lower-Frequency Absorption. Materials 2018, 11, 210. [CrossRef] [PubMed]

3. Watts, C.M.; Liu, X.; Padilla, W.J. Metamaterial electromagnetic wave absorbers. Adv. Mater. 2012, 24, OP98-OP120. [CrossRef] [PubMed]

4. Shen, G.; Zhang, M.; Ji, Y.; Huang, W.; Yu, H.; Shi, J. Broadband terahertz metamaterial absorber based on simple multi-ring structures. AIP Adv. 2018, 8, 075206. [CrossRef]

5. Landy, N.I.; Bingham, C.M.; Tyler, T.; Jokerst, N.; Smith, D.R.; Padilla, W.J. Design, theory, and measurement of a polarization-insensitive absorber for terahertz imaging. Phys. Rev. B 2009, 79, 125104. [CrossRef]

6. Ding, F.; Dai, J.; Chen, Y.; Zhu, J.; Jin, Y.; Bozhevolnyi, S.I. Broadband near-infrared metamaterial absorbers utilizing highly lossy metals. Sci. Rep. 2016, 6, 39445. [CrossRef]

7. Liu, N.; Mesch, M.; Weiss, T.; Hentschel, M.; Giessen, H. Infrared perfect absorber and its application as plasmonic sensor. Nano Lett. 2010, 10, 2342-2348. [CrossRef] 
8. Aydin, K.; Ferry, V.E.; Briggs, R.M.; Atwater, H.A. Broadband polarization-independent resonant light absorption using ultrathin plasmonic super absorbers. Nat. Commun. 2011, 2, 517. [CrossRef]

9. Wu, D.; Li, R.; Liu, Y.; Yu, Z.; Yu, L.; Chen, L.; Liu, C.; Ma, R.; Ye, H. Ultra-narrow Band Perfect Absorber and Its Application as Plasmonic Sensor in the Visible Region. Nanoscale Res. Lett. 2017, 12, 427. [CrossRef]

10. Callewaert, F.; Chen, S.; Butun, S.; Aydin, K. Narrow band absorber based on a dielectric nanodisk array on silver film. J. Opt. 2016, 18, 075006. [CrossRef]

11. Ye, H.; Wang, H.; Cai, Q. Two-dimensional VO2 photonic crystal selective emitter. J. Quant. Spectrosc. Radiat. Transf. 2015, 158, 119-126. [CrossRef]

12. Brückl, H.; Maier, T. Wavelength-tunable microbolometers with metamaterial absorbers. Opt. Lett. 2009, 34, 3012-3014.

13. Cui, Y.; He, Y.; Jin, Y.; Ding, F.; Yang, L.; Ye, Y.; Zhong, S.; Lin, Y.; He, S. Plasmonic and metamaterial structures as electromagnetic absorbers. Laser Photonics Rev. 2014, 8, 495-520. [CrossRef]

14. Shen, Y.; Zhou, J.; Liu, T.; Tao, Y.; Jiang, R.; Liu, M.; Xiao, G.; Zhu, J.; Zhou, Z.K.; Wang, X.; et al. Plasmonic gold mushroom arrays with refractive index sensing figures of merit approaching the theoretical limit. Nat. Commun. 2013, 4, 2381. [CrossRef] [PubMed]

15. Cetin, A.E.; Altug, H. Fano Resonant Ring/Disk Plasmonic Nanocavities on Conducting Substrates for Advanced Biosensing. ACS Nano 2012, 6, 9989-9995. [CrossRef] [PubMed]

16. Le, K.Q.; Ngo, Q.M.; Nguyen, T.K. Nanostructured Metal-Insulator-Metal Metamaterials for Refractive Index Biosensing Applications: Design, Fabrication, and Characterization. IEEE J. Sel. Top. Quantum Electron. 2017, 23, 388-393. [CrossRef]

17. Kabashin, A.V.; Evans, P.; Pastkovsky, S.; Hendren, W.; Wurtz, G.A.; Atkinson, R.; Pollard, R.; Podolskiy, V.A.; Zayats, A.V. Plasmonic nanorod metamaterials for biosensing. Nat. Mater. 2009, 8, 867-871. [CrossRef]

18. Zhou, L.; Tan, Y.; Wang, J.; Xu, W.; Yuan, Y.; Cai, W.; Zhu, S.; Zhu, J. 3D self-assembly of aluminium nanoparticles for plasmon-enhanced solar desalination. Nat. Photonics 2016, 10, 393-398. [CrossRef]

19. Lu, X.; Zhang, T.; Wan, R.; Xu, Y.; Zhao, C.; Guo, S. Numerical investigation of narrowband infrared absorber and sensor based on dielectric-metal metasurface. Opt. Express 2018, 26, 10179-10187. [CrossRef]

20. Liu, Y.; Gu, S.; Luo, C.; Zhao, X. Ultra-thin broadband metamaterial absorber. Opt. Express 2012, 108, 19-24. [CrossRef]

21. Zhou, P.; Zheng, G.G.; Xu, L.H.; Xian, F.L.; Lai, M. Broad-band and polarization-independent perfect absorption in graphene-gold cylinder arrays at visible and near-infrared wavelengths. Opt. Mater. 2018, 81, 59-63. [CrossRef]

22. Lee, D.; Han, S.Y.; Jeong, Y.; Nguyen, D.M.; Yoon, G.; Mun, J.; Chae, J.; Lee, J.H.; Ok, J.G.; Jung, G.Y.; et al. Polarization-sensitive tunable absorber in visible and near-infrared regimes. Sci. Rep. 2018, 8, 12393. [CrossRef] [PubMed]

23. Hulkkonen, H.; Sah, A.; Niemi, T. All-Metal Broadband Optical Absorbers Based on Block Copolymer Nanolithography. ACS Appl. Mater. Interfaces 2018, 10, 42941-42947. [CrossRef] [PubMed]

24. Liu, Z.Q.; Shao, H.B.; Liu, G.Q.; Liu, X.S.; Zhou, H.Q.; Hu, Y.; Zhang, X.N.; Cai, Z.J.; Gu, G. $\lambda 3 / 20000$ plasmonic nanocavities with multispectral ultra-narrowband absorption for high-quality sensing. Appl. Phys. Lett. 2014, 104, 081116. [CrossRef]

25. Odebo Lank, N.; Verre, R.; Johansson, P.; Kall, M. Large-Scale Silicon Nanophotonic Metasurfaces with Polarization Independent Near-Perfect Absorption. Nano Lett. 2017, 17, 3054-3060. [CrossRef] [PubMed]

26. Li, Z.; Butun, S.; Aydin, K. Ultranarrow Band Absorbers Based on Surface Lattice Resonances in Nanostructured Metal Surfaces. ACS Nano 2014, 8, 8242-8248. [CrossRef]

27. Yu, P.; Besteiro, L.V.; Huang, Y.; Wu, J.; Fu, L.; Tan, H.H.; Jagadish, C.; Wiederrecht, G.P.; Govorov, A.O.; Wang, Z. Broadband Metamaterial Absorbers. Adv. Opt. Mater. 2018, 7, 1800995. [CrossRef]

28. Miyazaki, H.T.; Kasaya, T.; Iwanaga, M.; Choi, B.; Sugimoto, Y.; Sakoda, K. Dual-band infrared metasurface thermal emitter for $\mathrm{CO}_{2}$ sensing. Appl. Phys. Lett. 2014, 105, 121107. [CrossRef]

29. Ma, W.; Wen, Y.; Yu, X. Broadband metamaterial absorber at mid-infrared using multiplexed cross resonators. Opt. Express 2013, 21, 30724-30730. [CrossRef]

30. Walter, R.; Tittl, A.; Berrier, A.; Sterl, F.; Weiss, T.; Giessen, H. Large-Area Low-Cost Tunable Plasmonic Perfect Absorber in the Near Infrared by Colloidal Etching Lithography. Adv. Opt. Mater. 2015, 3, $398-403$. [CrossRef] 
31. Cui, Y.; Fung, K.H.; Xu, J.; Ma, H.; Jin, Y.; He, S.; Fang, N.X. Ultrabroadband light absorption by a sawtooth anisotropic metamaterial slab. Nano Lett. 2012, 12, 1443-1447. [CrossRef] [PubMed]

32. Ji, D.; Song, H.; Zeng, X.; Hu, H.; Liu, K.; Zhang, N.; Gan, Q. Broadband absorption engineering of hyperbolic metafilm patterns. Sci. Rep. 2014, 4, 4498. [CrossRef] [PubMed]

33. Zhou, J.; Kaplan, A.F.; Chen, L.; Guo, L.J. Experiment and Theory of the Broadband Absorption by a Tapered Hyperbolic Metamaterial Array. ACS Photonics 2014, 1, 618-624. [CrossRef]

34. Wang, H.; Wang, L. Perfect selective metamaterial solar absorbers. Opt. Express 2013, 21 (Suppl. 6), A1078-A1093. [CrossRef]

35. Wang, H.; Prasad Sivan, V.; Mitchell, A.; Rosengarten, G.; Phelan, P.; Wang, L. Highly efficient selective metamaterial absorber for high-temperature solar thermal energy harvesting. Sol. Energy Mater. Sol. Cells 2015, 137, 235-242. [CrossRef]

36. Lin, L.; Zheng, Y. Optimizing plasmonic nanoantennas via coordinated multiple coupling. Sci. Rep. 2015, 5, 14788. [CrossRef]

37. Palik, E.D. Handbook of Optical Constants of Solids; Academic Press: Cambridge, MA, USA, 1985.

38. Cao, C.; Cheng, Y. A broadband plasmonic light absorber based on a tungsten meander-ring-resonator in visible region. Appl. Phys. A 2018, 125, 15. [CrossRef]

39. Yong, Z.; Zhang, S.; Gong, C.; He, S. Narrow band perfect absorber for maximum localized magnetic and electric field enhancement and sensing applications. Sci. Rep. 2016, 6, 24063. [CrossRef]

40. Hao, J.; Zhou, L.; Qiu, M. Nearly total absorption of light and heat generation by plasmonic metamaterials. Phys. Rev. B 2011, 83, 165107. [CrossRef]

41. Hagen, E.; Rubens, H. Über Beziehungen des Reflexions-und Emissionsvermögens der Metalle zu ihrem elektrischen Leitvermögen. Annalen der Physik 1903, 316, 873-901. [CrossRef]

42. Born, M.; Wolf, E. Principles of Optics: Electromagnetic Theory of Propagation, Interference and Diffraction of Light; Elsevier: Amsterdam, The Netherlands, 2013.

43. Sommerfeld, A. Lectures on Theoretical Physics Optics; Academic Press: New York, NY, USA, 1964 ; Volume 4.

44. Wenshan, C.; Shalaev, V. Optical Metamaterials. Fundamentals and Applications; Springer: New York, NY, USA, 2010; Volume 3, pp. 1-190.

(C) 2019 by the authors. Licensee MDPI, Basel, Switzerland. This article is an open access article distributed under the terms and conditions of the Creative Commons Attribution (CC BY) license (http://creativecommons.org/licenses/by/4.0/). 